\title{
PERFIL DA PESQUISA EM FINANÇAS NO BRASIL
}

\section{RESUMO}

Este trabalho analisa uma amostra de 551 artigos da área de finanças publicados entre 1974 e 2001 na Revista de Administração Contemporânea (RAC), Revista de Administração de Empresas (RAE), Revista de Administração da Universidade de São Paulo (RAUSP), Revista Brasileira de Economia (RBE) e Revista Brasileira de Mercado de Capitais (RBMEC), além dos 264 artigos incluídos dos Anais do Enanpad. O levantamento mostra que a maioria dos artigos apresenta somente um autor. A produtividade dos autores nacionais está mais concentrada em poucos indivíduos e é mais baixa do que o sugerido pela teoria bibliométrica. Mais de 70\% dos autores publicou apenas um artigo. Os artigos dos autores que publicaram pelo menos 4 trabalhos têm idade média maior do que a de artigos de autores igualmente prolíficos nos Anais do Enanpad. A maioria dos artigos são de autores afiliados à UFRJ, PUC/RJ e USP. Os autores do antigo Ibmec $^{1}$ aparecem em número expressivo, mas seus artigos estão concentrados na RBMEC, nas décadas de 70 e 80. Os autores da UFRGS, UFRJ e PUC/RJ respondem por dois terços da produção dos autores com pelo menos 4 artigos publicados nos Anais. O Rio de Janeiro aparece como o estado com mais autores prolíficos e não há autores nestas condições em outras regiões que não a Sul e a Sudeste.

\section{Ricardo Pereira Câmara Leal \\ COPPEAD / UFRJ}

\section{Jefferson de Oliveira \\ REFER - Fundação Rede Ferroviária de Seguridade Social}

\section{Aline Feldman Soluri}

Mellon Brascan DTVM

\begin{abstract}
This article analyzes 551 finance articles published between 1974 and 2001 at a sample of Brazilian academic journals besides 264 articles that appeared in the proceedings of the ANPAD meetings. Most articles are single authored. Author productivity is more concentrated in fewer individuals and is lower than suggested by the bibliometric theory. Over 70 percent of the authors published one single article. The articles from authors that published 4 or more journal articles show a greater average age than the articles from equally prolific authors in the ANPAD proceedings. Most prolific journal article authors are affiliated to UFRJ, PUC/RJ and USP. Authors from the former Ibmec are well ranked but their articles are concentrated in their institution's own publication in the 1970's and 80's. Authors affiliated to UFRGS, UFRJ, and PUC/RJ produced two thirds of the articles from authors with 4 or more articles in the ANPAD proceedings.
\end{abstract}

PALAVRAS-CHAVE Finanças no Brasil, literatura de finanças, produtividade em finanças, periódicos de finanças, Enanpad. KEY WORDS Finance in Brazil, brazilian finance literature, finance academic productivity, brazilian finance periodicals, ENANPAD. 


\section{INTRODUÇÃo}

O objetivo desse artigo é traçar um perfil da pesquisa em finanças no Brasil nas últimas décadas. Pretendemos identificar os veículos mais freqüentes de publicação dos trabalhos, quem são e onde atuam os principais pesquisadores. Traçar o perfil de uma determinada área, nesse sentido, não é um fato novo. Em finanças, porém, não conhecemos um trabalho dessa natureza no País.

Outras áreas, todavia, já apresentaram desenvolvimento de pesquisa desse tipo. Em Marketing, por exemplo, o trabalho de Vieira (1998) procurou analisar o impacto das publicações nacionais e estrangeiras sobre os trabalhos apresentados no Encontro Anual da Associação Nacional dos Programas de Pós-Graduação em Administração (Enanpad), enquanto Froemming et al. (2000) examinam as características metodológicas de artigos de marketing. Azzoni (1998) verifica quais são os autores mais citados na área de economia nas revistas acadêmicas brasileiras. A produtividade dos departamentos de economia e dos economistas brasileiros é analisada por Faria (2000). Bertero e Keinert (1994) analisam a evolução da literatura de organizações no Brasil, enquanto Vergara e Carvalho (1995) identificam a origem nacional dos autores dessa área. Bignetti e Paiva (2002) estudam as linhas de pensamento em estratégia dominantes nas citações de artigos brasileiros publicados nos Anais do Enanpad e listam nominalmente os autores nacionais e estrangeiros mais citados, concluindo que os autores nacionais são pouco mencionados. Já nos EUA, há vários trabalhos que analisam o perfil, o impacto e a qualidade dos periódicos da área de finanças, entre eles podemos citar Alexander e Mabry (1994), Borokhovich et al. (1994), Chung e Cox (1990), Mitenko e Diamond (1994), Zivney (1992), Zivney et al. (1994) e Zivney e Richenstein (1994). Isso se deve, em grande parte, ao fato de que, o processo de promoção do professor na carreira acadêmica e o processo de avaliação dos pro- gramas de pós-graduação por agentes externos indicam a necessidade de se conhecer o impacto e a qualidade da veiculação dos periódicos nesse país. Esse fato vem se intensificando também no Brasil nos últimos anos.

O presente trabalho busca, assim, preencher a lacuna formada pela falta de levantamento sobre a produção científica em finanças no Brasil.

\section{AMOSTRA}

Para elaborar este artigo, foi criado um banco de dados contendo a citação completa de trabalhos publicados que foram classificados como pertencentes à área de finanças ${ }^{2}$. A classificação da área a qual pertence o artigo foi feita pelos autores deste estudo e, portanto, como qualquer o critério de classificação, está sujeito à subjetividade. A necessidade de fazer essa classificação em artigos como de finanças se deu porque não há, no País, um periódico que seja exclusivamente da área e tenha publicação regular. Mesmo a RBMEC, que foi nitidamente a revista com maior conteúdo financeiro, publicou artigos que pertencem a outras áreas.

Para se formar o banco de dados, o acervo de bibliotecas do Rio de Janeiro e de São Paulo foi pesquisado e todos os exemplares existentes no acervo foram selecionados. As publicações selecionadas e seus respectivos anos de publicação estão na Tabela 1 .

O período de análise compreende os anos de 1974 a 2001 para que se obtenha 25 anos de consulta. Foram selecionadas as três principais revistas da área de Administração (RAUSP, RAE e RAC), segundo classificação Qualis da CAPES. A RBMEC, apesar de sua publicação irregular nos anos 80 e 90 e subseqüente extinção, foi um dos principais veículos de divulgação dos trabalhos acadêmicos de finanças no País durante muitos anos e, por isso, foi incluída em nossa análise. Ela utilizava um comitê editorial, com um ocasional processo de blind

Tabela 1 - Publicações selecionadas e ano de publicação

\begin{tabular}{|l|l|}
\hline \multicolumn{1}{|c|}{ PUBLICAÇÃO } & \multicolumn{1}{c|}{ ANO DE PUBLICAÇÃ0* } \\
\hline Anais do Enanpad & 1980 a 1982, 1984 a 1985, 1987 a 2001 \\
\hline Revista de Administração Contemporânea (RAC) & 1997 a 12/2001 \\
\hline Revista de Administração de Empresas (RAE) & 1974 a 12/2001 \\
\hline Revista de Administração da USP (RAUSP) & 1977 a 9/2001 \\
\hline Revista Brasileira de Economia (RBE) & 1974 a 12/2001 \\
\hline Revista Brasileira de Mercado de Capitais (RBMEC) & 1974 a 6/1996 \\
\hline
\end{tabular}

* Eventuais ausências de exemplares não são significativas e, portanto, não alteram de forma substancial o presente estudo. 
review, em particular nos últimos anos, segundo informações recebidas de um dos seus antigos editores, dada a sua importância passada e o seu caráter acadêmico, ela foi mantida na amostra. A RBE é uma revista de grande prestígio da área de economia que publica ocasionalmente trabalhos em finanças e, por isso, foi incluída. Uma revista de informação importante para a área, a Resenha BM\&F, não fará parte deste artigo porque, além de não utilizar blind review, não é um periódico referenciado e não tem, por finalidade, a divulgação de trabalhos acadêmicos, fugindo, assim, ao escopo do presente estudo. Os Anais do Enanpad também foram incluídos na análise porque representam o principal congresso da área de Administração do país. O primeiro Enanpad foi realizado em 1977. É possível que artigos publicados nos anais tenham versões posteriormente publicadas nos diversos periódicos escolhidos e, portanto, pode haver dupla contagem no total geral de artigos. Por exemplo, Zivney et al. (1994) sugerem que cerca de 30\% dos trabalhos apresentados no congresso da Eastern Finance Association dos EUA são publicados posteriormente em periódicos. Os artigos publicados nos Anais serão analisados separadamente por conta disso. Finalmente, cabe mencionar que os artigos dos Anais do Enanpad até 1997 incluíam uma pequena proporção de artigos de contabilidade (Ness Jr., 1998) que, de nenhuma forma, distorcem os resultados apresentados em nossa pesquisa e, portanto, não foram excluídos. Os anais dos três Enanpads em 1977, 1978 e 1979 não foram examinados diretamente mas Vasconcellos (1981) e Oliveira (1980) afirmam que os encontros de 1977 e 1978 trataram apenas de temas como o currículo de cursos de pós-graduação em Administração e que o encontro de 1979 teve trabalhos nas áreas de políticas públicas, administração de ciência e tecnologia, pequenas e médias empresas e em gerência de exportação. Assim, não há evidências de trabalhos em finanças nestes anais. Já os anais de 1983 e 1986 não puderam ser encontrados nas bibliotecas nacionais. Como o número de trabalhos de finanças publicados nessa época era pequeno, a omissão destes dois exemplares não irá alterar nossos resultados simultaneamente.

A seleção da amostra pode ter pecado por não incluir outras revistas das áreas de Administração, Economia, Pesquisa Operacional e Engenharia de Produção, que ocasionalmente trazem artigos da área de finanças. Outra falha possível deste artigo pode ser não incluir artigos de anais de congressos como o ENEGEP - Encontro Nacional de Engenharia de Produção, da SOBRAPO (Pesquisa Operacional), da Sociedade Brasileira de Econometria (SBE) ou da Sociedade Brasileira de Finanças, entre outros. Preferimos incluir somente o Enanpad porque é o que tem uma área de finanças constituída há mais tempo. É possível que os resultados apresentados a seguir sejam tendenciosos, revelando um perfil de grande parte do que foi publicado por autores atuantes predominantemente na área de Administração. De qualquer forma, apesar das limitações do nosso estudo, esperamos contribuir com um primeiro perfil da área no Brasil, que poderá ser aperfeiçoado e estudado com mais profundidade em trabalhos futuros. Dissertações e teses não serão levadas em consideração uma vez que o objetivo do presente artigo é analisar a publicação em finanças nos principais periódicos e congressos nacionais.

Foram analisados 815 artigos, sendo 264 dos Anais do Enanpad e 551 dos periódicos selecionados. A RBMEC lidera com 291 artigos selecionados. Ela também apresentou o maior número de artigos selecionados por exemplar entre os periódicos. Entretanto, não podemos comparar a RBMEC, voltada para o mercado financeiro, com outros periódicos que tratam também da publicação de artigos na área de economia e admi-

Tabela 2 - Número de artigos selecionados e exemplares selecionados por publicação

\begin{tabular}{|l|r|r|c|}
\hline \multicolumn{1}{|c|}{ PERIÓDICOS } & № DE ARTIGOS & № DE EXEMPLARES & ARTIGOS POR EXEMPLARES* \\
\hline Anais do Enanpad & 264 & 23 & 11,48 \\
\hline Revista de Administração Contemporânea (RAC) & 16 & 14 & 1,14 \\
\hline Revista de Administração de Empresas (RAE) & 112 & 127 & 0,88 \\
\hline Revista de Administração da USP (RAUSP) & 85 & 94 & 0,90 \\
\hline Revista Brasileira de Economia (RBE) & 47 & 119 & 0,39 \\
\hline Revista Brasileira de Mercado de Capitais (RBMEC) & 291 & 45 & 6,47 \\
\hline Total & 551 & & \\
\hline Total Revistas \& Enanpad & 815 & & \\
\hline
\end{tabular}


nistração como um todo, uma vez que levamos em conta somente trabalhos publicados em finanças. Os outros periódicos tiveram menos de um artigo selecionado por exemplar publicado, o que significa dizer que alguns exemplares não continham artigos de finanças. A RAC apareceu melhor entre os periódicos não especializados em finanças, com uma média de 1,14 artigo por fascículo. A Tabela 2 mostra a quantidade de artigos selecionados por publicação.

Nos Anais do Enanpad, verifica-se que entre os anos de 1982 até 1992 foram publicados poucos trabalhos. A partir do ano de 1993, a quantidade de artigos publicados aumentou significativamente em comparação aos anos anteriores. A RAE apresentou um número considerável de artigos publicados em finanças nos anos de 1974 a 1979, no entanto, a partir de 1980 a quantidade de artigos publicados por ano diminuiu. A RBMEC, a partir de 1985, apresentou uma queda na quantidade de artigos, comparada aos anos anteriores. Isso certamente se deve à sua publicação irregular a partir de

Tabela 3 - Quantidade de artigos por periódicos e ano de publicação*

\begin{tabular}{|c|c|c|c|c|c|c|}
\hline ANO & RAC & RAE & RAUSP & RBE & RBMEC & ANAIS \\
\hline 1974 & $\mathrm{NP}$ & $16(3)$ & NP & $1(1)$ & $6(1)$ & $\mathrm{NP}$ \\
\hline 1975 & NP & $5(4)$ & NP & $2(2)$ & $18(3)$ & NP \\
\hline 1976 & NP & $9(5)$ & NP & 1 (1) & $17(3)$ & NP \\
\hline 1977 & NP & $4(1)$ & 0 & 0 & $19(3)$ & 0 \\
\hline 1978 & NP & $3(3)$ & 0 & 0 & $19(3)$ & 0 \\
\hline 1979 & NP & $8(4)$ & $3(3)$ & $2(1)$ & $29(3)$ & 0 \\
\hline 1980 & NP & $3(3)$ & $5(4)$ & $2(1)$ & $35(3)$ & 0 \\
\hline 1981 & NP & $3(1)$ & $5(3)$ & $2(2)$ & $22(3)$ & 0 \\
\hline 1982 & NP & $3(3)$ & $1(1)$ & $5(4)$ & $21(3)$ & 2 \\
\hline 1983 & NP & $1(1)$ & $4(3)$ & $1(1)$ & $17(4)$ & ND \\
\hline 1984 & NP & $2(2)$ & $2(1)$ & 1 (1) & $19(4)$ & 0 \\
\hline 1985 & NP & $3(1)$ & $5(3)$ & $2(2)$ & $8(2)$ & 4 \\
\hline 1986 & NP & $2(2)$ & $1(1)$ & 0 & $4(1)$ & ND \\
\hline 1987 & NP & $2(1)$ & $3(1)$ & 0 & $11(2)$ & 12 \\
\hline 1988 & NP & $2(1)$ & $2(1)$ & 1 (1) & $5(1)$ & 7 \\
\hline 1989 & NP & $1(1)$ & $3(2)$ & 1 (1) & 0 & 14 \\
\hline 1990 & NP & $3(2)$ & 1 (1) & $2(2)$ & $4(1)$ & 8 \\
\hline 1991 & NP & $3(3)$ & $5(3)$ & $1(1)$ & $9(2)$ & 11 \\
\hline 1992 & NP & $4(2)$ & $4(3)$ & 1 (1) & $7(2)$ & 11 \\
\hline 1993 & NP & $2(1)$ & $4(3)$ & $2(1)$ & $5(1)$ & 20 \\
\hline 1994 & NP & $4(4)$ & $4(3)$ & $2(2)$ & $8(2)$ & 21 \\
\hline 1995 & $\mathrm{NP}$ & $3(3)$ & 0 & 1 (1) & $5(2)$ & 21 \\
\hline 1996 & NP & $5(4)$ & $4(2)$ & 1 (1) & $3(1)$ & 23 \\
\hline 1997 & $3(2)$ & $5(4)$ & $5(2)$ & $4(3)$ & NP & 25 \\
\hline 1998 & $3(3)$ & $4(4)$ & $4(2)$ & $2(2)$ & NP & 18 \\
\hline 1999 & $2(2)$ & $3(3)$ & $4(2)$ & $2(2)$ & NP & 20 \\
\hline 2000 & $3(2)$ & $5(4)$ & $5(2)$ & $5(3)$ & NP & 27 \\
\hline 2001 & $5(3)$ & $4(2)$ & $11(3)$ & $4(3)$ & NP & 20 \\
\hline Total & 16 & 112 & 85 & 47 & 291 & 264 \\
\hline
\end{tabular}

*Os números entre parênteses representam a quantidade de exemplares com artigos de finanças. Os anais do ENANPAD são publicados uma vez ao ano. "ND" significa "não disponível" nos acervos consultados e "ND" significa "não publicado". 
meados dos anos 80. Os demais periódicos não apresentaram um padrão diferenciado no tempo.

A Tabela 3 detalha a amostra para cada ano.

\section{ANÁLISE QUANTITATIVA DA AUTORIA DOS ARTIGOS}

Verificaremos, a seguir, que há um número expressivo de trabalhos com um único autor e uma quantidade bem menor de trabalhos com dois autores. Nota-se que nos Anais do Enanpad é mais comum se observar 3 ou mais autores. Isso pode indicar a ocorrência mais freqüente de trabalhos de alunos no congresso do que nos periódicos. Muitos desses podem acabar não sendo publicados nos periódicos. Entretanto, isso não foi analisado neste artigo. A ocorrência de autor único na área de finanças dos Anais do Enanpad é mais freqüente do que na área de marketing pesquisada por Vieira (1998). Já nos periódicos, a ocorrência de autor único é ainda mais freqüente do que nos anais. Os artigos com apenas um autor respondem por $70 \%$ do total. A Tabela 4 analisa a quantidade de autores por artigo e a quantidade de artigos por autor. Os percentuais do total estão entre parêntesis.

Analisando a literatura internacional, verificamos Lotka, segundo Chung e Cox (1990), apresenta uma lei que diz que o número de autores que publica $n$ artigos é igual a $1 / \mathrm{n}^{2}$ dos autores que publicam apenas um artigo. Assim, o número de autores que publicam dois artigos é igual a 1/4 do número de autores que publicam um artigo, o número de autores que publicam 3 artigos é igual a 1/9 dos que publicam um artigo, e assim por diante. Ainda segundo Lotka, que pesquisou o padrão de publicações em química e física, o número de autores com uma única publicação é de cerca de $60 \%$. Além disso, sua lei traduz a lógica de que quem publica mais tem maior probabilidade de continuar publicando do que quem não publica muito. Chung e Cox (1990) demonstram que o número de autores com um único trabalho publicado, segundo a lei de Lotka, deveria ser de $6 / \pi^{2}$ que resulta numa constante igual a $60,8 \%$ dos autores. A partir daí, o número de autores com dois trabalhos publicados seria $1 / 4$ dessa frequência, ou 15,2\%, o número de autores com 3 trabalhos publicados seria de $1 / 9$ dessa freqüência, ou $6,8 \%$ e assim por diante.

De acordo com as freqüências teóricas calculadas pela Lei de Lotka, fica evidente que a ocorrência de autores que publicam somente uma vez em finanças no Brasil é muito maior do que a frequência teórica de $60,8 \%$. O percentual de autores com 2 ou mais trabalhos é sempre menor do que o previsto por Lotka e fica cada vez menor conforme o número de artigos aumenta.

A Tabela 5 mostra o número de autores segundo a quantidade de artigos que publicaram. Os percentuais em relação ao total de artigos publicados em finanças em cada periódico aparecem em parêntesis. Eles podem não somar 100\% devido aos arredondamentos. Foi estimada a regressão passando pela origem $\log \left(a_{n} / a_{1}\right)=-c x$ $\log (\mathrm{n})$, onde $\mathrm{a}_{1}$ é o número de autores com 1 artigo, $\mathrm{a}_{\mathrm{n}} \mathrm{o}$ número de autores com $n$ artigos, $n$ é o número de artigos e c é o coeficiente generalizado para a Lei de Lotka. A Lei de Lotka diz que $a_{n}=a_{1} / n^{2}$. Os valores padrão da Lei de Lotka indicando o percentual de autores pela quantidade de artigos publicados aparecem na coluna mais à direita na tabela. A Lei de Lotka generalizada é $a_{n}$ $=a_{1} / n^{c}$. Os valores estimados para $c$ para cada veículo de publicação bem como o $\mathrm{R}^{2}$ da regressão estão na tabela. Um valor de $c$ maior do que 2 indica um número menor de autores com mais de um artigo publicado do que o previsto pela referida lei. O teste de qui-quadrado foi realizado para até 5 artigos, para se desconsiderar possíveis valores extremos. O valor crítico a $5 \%$ de significância é de 9,49, indicando que o coeficiente é

Tabela 4 - Número de autores por artigo

\begin{tabular}{|c|r|r|r|r|r|r|r|}
\hline NNo DE AUTORES & \multicolumn{1}{|c|}{ RAC } & \multicolumn{1}{|c|}{ RAE } & \multicolumn{1}{|c|}{ RAUSP } & \multicolumn{1}{|c|}{ RBE } & \multicolumn{1}{|c|}{ RBMEC } & TOTAL DE PERIÓdICOS & ANAIS ENANPAD \\
\hline 1 & $4(25)$ & $79(70,5)$ & $44(51,8)$ & $27(57,4)$ & $230(79)$ & $384(69,7)$ & $121(45,8)$ \\
\hline 2 & $10(62,5)$ & $27(24,1)$ & $36(42,4)$ & $16(34)$ & $55(18,9)$ & $144(26,1))$ & $97(36,7)$ \\
\hline 3 & $2(12,5)$ & $6(5,4)$ & $4(4,7)$ & $2(4,3)$ & $6(2,1)$ & $20(3,6)$ & $38(14,4)$ \\
\hline 4 & 0 & 0 & $1(1,2)$ & $1(2,1)$ & 0 & $2(0,4)$ & $6(2,3)$ \\
\hline 5 & 0 & 0 & 0 & $1(2,1)$ & 0 & $1(0,2)$ & $1(0,4)$ \\
\hline 6 & 0 & 0 & 0 & 0 & 0 & 0 & $1(0,4)$ \\
\hline Total & $16(100)$ & $112(100)$ & $85(100)$ & $47(100)$ & $291(100)$ & $551(100)$ & $264(100)$ \\
\hline
\end{tabular}


diferente de zero a um nível de significância de 5\%, no caso de $c$, e que o coeficiente qui-quadrado é significativo. "NA" significa "não se aplica".

Chung e Cox (1990) apresentam um número de 62\% de autores que publicaram somente uma vez para uma amostra dos principais periódicos internacionais da área de finanças. A Tabela 5 sugere que a produtividade dos autores nacionais de finanças é mais baixa que a dos autores internacionais e menor que a produtividade teórica derivada de experiência de várias disciplinas. Um número muito elevado de autores publicou somente um artigo nos periódicos analisados. Isso pode ser atribuído a um elevado número de trabalhos de alunos que ingressam no mercado profissional e não tornam a publicar nos meios acadêmicos. Seria interessante comparar esta questão com outras áreas afins. Infelizmente, Faria (2000) somente examina a produtividade em termos de publicações internacionais na área de economia no Brasil. Suas conclusões são consistentes com os resultados aqui apresentados: a produtividade dos depar- tamentos de economia brasileiros é baixa em relação aos seus congêneres internacionais e o sucesso de publicação está concentrado em poucos indivíduos, mesmo nos departamentos de maior prestígio. Vieira (1998) não examina esta questão explicitamente para a área de marketing do Enanpad, mas sua informação de que somente 12 autores são responsáveis por cerca de 40\% das publicações é sugestiva e aplicável à área de finanças também. Outra inferência poderia ser feita a partir do número de autores com 4 trabalhos em marketing publicados nos Anais do Enanpad até 1997: segundo Vieira (1998), há seis autores com 4 trabalhos publicados, que corresponderiam à freqüência teórica de Lotka de $3,8 \%$. Sabendo que a freqüência teórica de Lotka para autores com 1 trabalho é de 60,8\%, uma regra de três simples indica que deveria haver 96 autores na área de marketing do Enanpad que publicaram somente um trabalho. Considerando que o número de autores até 2001 com um só trabalho na área de finanças do Enanpad era de 202 e que o número de trabalhos apre-

Tabela 5 - Número de autores por quantidade de artigos publicados, separados por periódico

\begin{tabular}{|c|c|c|c|c|c|c|c|c|}
\hline $\begin{array}{l}\text { № DE } \\
\text { ARTIGOS }\end{array}$ & RAC & RAE & RAUSP & RBE & RBMEC & $\begin{array}{l}\text { ANAIS DO } \\
\text { ENANPAD }\end{array}$ & $\begin{array}{c}\text { TODAS AS } \\
\text { PUBLICAÇÕES }\end{array}$ & $\begin{array}{c}\% \text { LOTKA } \\
\text { C }=2\end{array}$ \\
\hline 1 & $18(81,8)$ & $94(80,3)$ & $77(83,7)$ & $61(92,4)$ & $142(71,4)$ & $209(74,9)$ & $601(77,5)$ & 60,80 \\
\hline 2 & $2(9,1)$ & $14(12,0)$ & $5(5,4)$ & $4(6,1)$ & $23(11,6)$ & $38(13,6)$ & $86(11,1)$ & 15,20 \\
\hline 3 & $1(4,5)$ & $7(6,0)$ & $5(5,4)$ & 0 & $15(7,5)$ & $15(5,4)$ & $43(5,5)$ & 6,80 \\
\hline 4 & 0 & $1(0,9)$ & $1(1,1)$ & 0 & $7(3,5)$ & $6(2,2)$ & $14(1,9)$ & 3,80 \\
\hline 5 & $1(4,5)$ & $1(0,9)$ & $1(1,1)$ & $1(1,5)$ & $4(2,0)$ & $1(0,4)$ & $9(1,2)$ & 2,43 \\
\hline 6 & 0 & 0 & $1(1,1)$ & 0 & $2(1,0)$ & $3(1,1)$ & $6(0,8)$ & 1,69 \\
\hline 7 & 0 & 0 & $1(1,1)$ & 0 & $1(0,5)$ & $1(0,4)$ & $3(0,4)$ & 1,24 \\
\hline 8 & 0 & 0 & $1(1,1)$ & 0 & 0 & $1(0,4)$ & $2(0,3)$ & 0,95 \\
\hline 9 & 0 & 0 & 0 & 0 & 0 & $1(0,4)$ & $1(0,1)$ & 0,75 \\
\hline 10 & 0 & 0 & 0 & 0 & $2(1,0)$ & 0 & $2(0,3)$ & 0,61 \\
\hline 11 & 0 & 0 & 0 & 0 & $2(1,0)$ & $1(0,4)$ & $3(0,4)$ & 0,50 \\
\hline$\ldots$ & ... & $\cdots$ & $\cdots$ & $\cdots$ & $\cdots$ & $\ldots$ & $\ldots$ & $\ldots$ \\
\hline 14 & 0 & 0 & 0 & 0 & 0 & $1(0,4)$ & $1(0,1)$ & 0,31 \\
\hline 15 & 0 & 0 & 0 & 0 & $1(0,5)$ & 0 & $1(0,1)$ & 0,27 \\
\hline 16 & 0 & 0 & 0 & 0 & 0 & $1(0,4)$ & $1(0,1)$ & 0,24 \\
\hline$\ldots$ & ... & $\cdots$ & $\ldots$ & $\ldots$ & ... & $\ldots$ & $\ldots$ & $\ldots$ \\
\hline 22 & 0 & 0 & 0 & 0 & 0 & $1(0,4)$ & $1(0,1)$ & 0,13 \\
\hline Total & $22(100)$ & $117(100)$ & 92 (100) & $66(100)$ & $199(100)$ & $279(100)$ & 775 (100) & 100,00 \\
\hline c & $2,19 *$ & $2,87^{*}$ & $2,46^{*}$ & $2,77^{*}$ & $2,09 *$ & $2,23 *$ & $2,44^{*}$ & NA \\
\hline $\mathbf{R}^{2}$ & 0,47 & 0,71 & 0,69 & 0,41 & 0,81 & 0,72 & 0,93 & NA \\
\hline$\chi^{2}$ & 3,54 & $12,13^{*}$ & $17,54 *$ & $21,68^{*}$ & 7,46 & $19,84^{*}$ & $65,30 *$ & NA \\
\hline
\end{tabular}


sentados no Enanpad é maior na área de marketing do que na área de finanças ${ }^{3}$, o número real de autores com somente um trabalho em marketing deve ser muito maior do que 96, sugerindo que a frequêencia de autores com somente um trabalho publicado em marketing é muito maior do que a frequência teórica. Admitindo que tal ocorrência possa ser observada também na área de finanças, é bem provável que o fenômeno do excessivo número de autores com somente um trabalho publicado nessa área no Brasil não seja um privilégio. Pelo contrário, não seria de todo surpreendente que as conclusões de Faria (2000) para a área de economia, uma área estabelecida há mais tempo e com programas considerados como de nível internacional pela CAPES, se aplicassem à área de finanças.

Segundo Powers et al. (1998), cerca de metade daqueles que se doutoram em marketing nos EUA jamais publicaram um artigo sequer nos periódicos de marketing, e grande parte dos que publicaram o fizeram somente uma vez. É claro que é possível que esses autores tenham se utilizado de outros veículos para publicar, mas Powers et al. (1998) analisaram um grande número de periódicos da área de marketing. A quantidade de acadêmicos que publicam pouco não deixa de ser surpreendente considerando a pressão crescente sobre a produção acadêmica para a progressão na carreira e para a avaliação dos programas de pós-graduação.

A significância estatística do que se sugere acima foi examinada por meio de um teste qui-quadrado. Os resultados na Tabela 5 indicam que as freqüências observadas para o número de artigos por autor para os veículos de publicação brasileiros examinados são estatisticamente diferentes dos valores teóricos esperados pela Lei de Lotka. Há duas exceções: a RAC e a RBMEC. Para essas duas revistas, os números estão dentro do previsto. Para as demais revistas e para os Anais do Enanpad, fica claro que o número de autores com mais de um artigo publicado é menor do que o previsto pela Lei de Lotka. Estes resultados são consistentes com o quadro de baixa produtividade dos autores da área.

Finalmente, é possível estimar quais são os expoentes adequados para a redução do número de autores com mais de um artigo a partir de uma Lei de Lotka generalizada. Ela diz que:

$$
a_{n}=a_{1} \times \frac{1}{n^{2}}
$$

onde $a_{n}$ é o número de autores que publicaram $n$ artigos, $a_{1}$ é o número de autores que publicaram 1 artigo e $n$ é o número de artigos em questão. Uma generaliza- ção poderia ser escrita, considerando um coeficiente genérico $c$ em lugar do quadrado do número de artigos. Assim, a Lei de Lotka genérica seria expressa por:

$$
a_{n}=a_{1} \times \frac{1}{n^{c}}
$$

Seria possível, então, estimar o coeficiente $c$ para cada periódico ou amostra de periódicos de interesse a partir das frequências observadas e comparar o coeficiente estimado com o coeficiente padrão de Lotka que é igual a 2. Caso o coeficiente estimado seja maior do que 2, haverá indicação de que o número de autores que produz mais do que um artigo é menor do que o previsto pela referida lei. Caso contrário, o número será maior. Pode-se estimar o coeficiente $c$ por meio da seguinte regressão linear, forçando-se a reta ajustada a passar pela origem:

$$
\log \left(a_{n} / a_{1}\right)=-c \times \log (n)
$$

Todos os coeficientes estimados são significativamente maiores do que zero na Tabela 5 mas apenas os da RAUSP e os da RAE são significativamente diferentes e maiores do que 2. Para essas revistas, o número de autores que se espera que publiquem somente um artigo está acima do previsto por Lotka. A RAE aparece com o coeficiente mais alto. A RBE tem o segundo maior coeficiente mas, devido ao seu elevado erro padrão, ele não é significativamente diferente de 2. Estas revistas, notadamente seletivas, têm um número muito elevado de autores com somente um artigo de finanças nela publicado. A RAUSP aparece como o veículo do meio de administração com o maior percentual de autores com somente um artigo. Tais resultados podem indicar que a RAE e a RBE são as mais seletivas na área ou podem apenas indicar que o número de autores brasileiros que publicam somente um artigo é maior do que o previsto pela teoria bibliométrica. Acreditamos que todas as revistas podem ser igualmente seletivas uma vez que a RAC apresenta uma amostra pequena devido à sua curta existência e, portanto, não se pode descartar que ela não seja tão seletiva quanto as demais. A RBMEC, de outra forma, não pode ser comparada com as demais porque não utilizou o procedimento de blind review durante a maior parte de sua existência, além de ter sido o veículo preferencial para a divulgação dos trabalhos realizados pelo Ibmec no passado. A RBE e a RAE são as revistas, entre as que analisamos, que aparecem mais bem classificadas por Azzoni (2000), segundo citações recebidas por seus artigos no Brasil e no exterior. Talvez o comportamento da freqüen- 
cia de publicação dos autores em revistas nacionais possa ser previsto melhor com coeficientes maiores do que 2 , como os que constam da Tabela 5.

\section{PRODUTIVIDADE DOS AUTORES DE FINANÇAS NOS PERIÓDICOS}

Investigar quais são os autores mais produtivos é comum em finanças. Alexander e Mabry (1994), por exem- plo, examinam quem são os autores de finanças mais prolíficos e influentes, quais os periódicos que merecem mais citações e quais os artigos da área que tiveram mais impacto por meio das citações. O impacto de um número seleto de periódicos nas diferentes áreas de finanças é estudado por Borokhovich et al. (1994). Azzoni (1998) verifica quais são os artigos e os autores mais citados por área de especialidade em economia no Brasil. Faria (2000) examina a produtividade dos autores e dos programas de economia brasileiros por meio da

Tabela 6 - Autores que mais publicaram em periódicos nacionais selecionados

\begin{tabular}{|c|c|c|c|c|c|c|c|c|}
\hline AUTOR & $\begin{array}{l}\text { VÍNCULOS PROFISSIONAIS } \\
\text { NA ÉPOCA DA PUBLICAÇÃOO }\end{array}$ & $\begin{array}{c}\text { № DE } \\
\text { ARTIGOS }\end{array}$ & $\begin{array}{l}\text { IDADE MÉDIA } \\
\text { DOS ARTIGOS }\end{array}$ & RAC & RAE & RAUSP & RBE & RBMEC \\
\hline Ney Roberto Ottoni de Brito & UFRJ & 21 & 21,52 & 0 & 1 & 7 & 2 & 11 \\
\hline Clóvis de Faro & IPEA/RJ, FGV/RJ, UFRJ, UFF, UERJ & 18 & 19,89 & 0 & 2 & 1 & 5 & 10 \\
\hline Walter Lee Ness Júnior & IBMEC/RJ, PUC/RJ, NYU/EUA & 16 & 19,00 & 0 & 0 & 1 & 0 & 15 \\
\hline Antonio Zoratto Sanvicente & USP & 12 & 15,58 & 0 & 3 & 8 & 0 & 1 \\
\hline Jairo Laser Procianoy & UFRGS, USP & 12 & 7,25 & 0 & 5 & 6 & 0 & 1 \\
\hline Horacio de Mendonça Netto & IBMEC/RJ, CVM/RJ & 11 & 23,45 & 0 & 0 & 0 & 0 & 11 \\
\hline Miguel Dirceu Fonseca Tavares & IBMEC/RJ & 10 & 21,40 & 0 & 0 & 0 & 0 & 10 \\
\hline Newton C. A. da Costa Júnior & UFSC, U. Lancaster/GB & 10 & 4,70 & 2 & 2 & 3 & 2 & 1 \\
\hline Ricardo Pereira Câmara Leal & $\begin{array}{l}\text { UFRJ, PUC/RJ, UNR/EUA, } \\
\text { Georgetown/EUA }\end{array}$ & 10 & 7,10 & 1 & 1 & 5 & 0 & 3 \\
\hline Tara Keshar Nanda Baidya & PUC/RJ & 8 & 12,50 & 0 & 1 & 0 & 1 & 6 \\
\hline Claudio Roberto Contador & IPEA/RJ, UFRJ, IBMEC/RJ & 7 & 23,14 & 0 & 0 & 2 & 1 & 4 \\
\hline Luiz Augusto de Bragança & IBMEC/RJ, U. Chicago/EUA & 7 & 20,00 & 0 & 0 & 0 & 0 & 7 \\
\hline Marcos Fernandes Machado & IBMEC/RJ & 6 & 20,83 & 0 & 0 & 0 & 0 & 6 \\
\hline Arnoldo Wald & UERJ, PUC/RJ & 5 & 24,00 & 0 & 0 & 0 & 0 & 5 \\
\hline Domingos de Gouveia Rodrigues & IBMEC/RJ & 5 & 18,00 & 0 & 0 & 0 & 0 & 5 \\
\hline Jaime Evaldo Fensterseifer & UFRGS, UCLA/EUA & 5 & 13,40 & 0 & 1 & 4 & 0 & 0 \\
\hline Manuel José da Rocha Armada & U. do Minho/Portugal & 5 & 3,60 & 5 & 0 & 0 & 0 & 0 \\
\hline Nelson Laks Eizirik & IBMEC/RJ, UCAM/RJ & 5 & 23,20 & 0 & 0 & 0 & 0 & 5 \\
\hline Reginaldo Ferreira Pereira & IBMEC/RJ, CVM/RJ & 5 & 22,20 & 0 & 0 & 0 & 0 & 5 \\
\hline Antonio Marcos Duarte Junior & $\begin{array}{l}\text { Unibanco, BBM, Princeton/EUA, } \\
\text { IMPA, PUC/RJ }\end{array}$ & 4 & 7,50 & 0 & 0 & 0 & 0 & 4 \\
\hline Carlos Patrício Samanez & PUC/RJ & 4 & 11,75 & 0 & 0 & 0 & 0 & 4 \\
\hline Henrique Goldkorn & Consultor independente & 4 & 26,75 & 0 & 4 & 0 & 0 & 0 \\
\hline Pedro Carvalho de Mello & IBMEC/RJ & 4 & 22,50 & 0 & 0 & 0 & 0 & 4 \\
\hline Paulo Sérgio Ceretta & UFSM, UFSC & 4 & 1,25 & 2 & 1 & 1 & 0 & 0 \\
\hline Roberto Marcos da Silva Montezano & IBMEC/RJ & 4 & 13,75 & 0 & 0 & 0 & 0 & 4 \\
\hline Rubens Famá & USP & 4 & 3,50 & 1 & 0 & 3 & 0 & 0 \\
\hline Ubiratan Jorge lorio de Souza & IBMEC/RJ, FINES & 4 & 17,75 & 0 & 0 & 0 & 0 & 4 \\
\hline
\end{tabular}


publicação em periódicos internacionais. Vieira (1998) pesquisa os autores e programas mais prolíficos na área de marketing no Brasil e o padrão de citações em marketing nos Anais do Enanpad. O impacto de se publicar em diferentes periódicos da área de finanças e as políticas editoriais desses periódicos são objeto de estudo de Zivney e Reichenstein (1994) e Mitenko e Diamond (1994). Finalmente, Koljatic e Silva (2001) observam o impacto das publicações internacionais de autores latino-americanos de economia e administração.

No Brasil, procuramos identificar, na área de finanças, quais são os autores mais prolíficos, qual o seu vínculo profissional e o ano em que publicaram. Na Tabela 6 estão os 27 autores que publicaram 4 artigos ou mais na amostra. Vários autores publicaram somente em um periódico, notadamente a RBMEC. Treze autores, quase metade dos autores mais prolíficos listados, publicaram somente na RBMEC. Há indícios de que a revista servia de veículo para a divulgação dos trabalhos de pesquisa do antigo Ibmec, e, como citamos anteriormente, os processos de blind review nesse periódico eram ocasionais. Isso de forma alguma desmerece a revista, mas sugere o papel da RBMEC como house organ. Os trabalhos dos autores do antigo Ibmec, entretanto, são os que têm idade média mais alta, refletindo o fato de que a maioria foi publicada nos anos 70 e no início dos anos 80. De todos os autores listados na tabela 6 , somente 7 apresentam idade média de seus artigos inferior a 10 anos. Estes devem estar entre os autores com maior potencial para continuar publicando. Na tabela, também há predominância de autores do estado do Rio de Janeiro por conta, em parte, dos artigos da RBMEC.

Fizemos um levantamento dos artigos internacionais de finanças em língua inglesa publicados pelos autores prolíficos que aparecem na tabela 6 por meio de pesquisa nos bancos de dados PROQUEST, EMERALD, ECONLIT e ECONBASE da Elsevier além de contatos diretos com os autores. O resultado do levantamento está no apêndice. A tabela mostra somente os autores que publicaram pelo menos 4 artigos. A idade média dos artigos foi calculada dividindo-se a idade em anos de cada artigo até o ano de 2001 pelo total de artigos publicados pelo autor. O total de autores é 27 e o total de artigos 210.

Muitos autores prolíficos não possuem publicações internacionais que apareçam nas fontes pesquisadas. $\mathrm{O}$ que se pode perceber pela análise do apêndice é que alguns dos autores publicaram em periódicos de grande reputação, como o Journal of Finance, há muito tempo. Há publicações recentes em periódicos de grande pres- tígio, mas a quantidade de publicações está concentrada em pouquíssimos autores, tal como foi observado por Faria (2000) para a área de economia.

Na Tabela 7, teremos uma análise do número de artigos por autores que publicaram 4 artigos ou mais na amostra segundo o seu vínculo institucional. O número de vínculos profissionais dos autores foi considerado para elaboração dessa tabela. Para os casos que apresentam mais de um vínculo por autor, dividimos um pelo número de vínculos para ratear a publicação entre as instituições. Como era esperado, os autores vinculados ao antigo Ibmec nos anos 70 e 80 aparecem em maior número. Seguem-se a PUC-RJ, UFRJ e USP com o número mais expressivo de autores, com pelo menos 4 artigos publicados em periódicos. A tabela também mostra que os autores vinculados ao antigo Ibmec publicaram prioritariamente na RBMEC. A tabela conta apenas os artigos de autores que publicaram 4 ou mais artigos na amostra. Foi atribuída uma fração igual do artigo para cada instituição quando o autor declara mais de um vínculo profissional.

Esse dados indicam claramente que os profissionais que trabalhavam como pesquisadores no instituto nos anos 70 e 80 consideravam a RBMEC como o único veículo de interesse para publicar seus artigos, até mesmo pelo fato de ser a única revista de cunho acadêmico especializada em finanças. Entretanto, tal fato certamente distorce a amostra. De um lado, a preferência dos autores pelas revistas de suas próprias instituições também fica caracterizada, em grau bem menor, nos casos da USP e FGV-EAESP. Por outro lado, a UFRJ, a UFRGS e a PUCRJ, que não têm revista própria, tendem a distribuir suas publicações entre as diversas revistas. Cabe observar que há também uma concentração dos trabalhos da PUC-RJ e da UFRJ na RBMEC.

É interessante observarmos a relação de artigos segundo o estado ou o país em que se localiza a instituição dos autores com 4 ou mais artigos publicados no país. O Rio de Janeiro representa 68\% dos artigos. Há várias explicações possíveis para essa liderança. Em primeiro lugar, obviamente, está o fato de o antigo Ibmec ter sido sediado nessa cidade. Entretanto, mesmo ao se desconsiderar os trabalhos do antigo Ibmec, outras instituições do Rio de Janeiro aparecem com destaque.

A USP responde por praticamente toda a produção paulista da amostra de autores com pelo menos 4 trabalhos publicados em periódicos. O Rio Grande do Sul aparece em quarto lugar depois dos autores de instituições localizadas nos EUA. Tais dados apresentam maior expressividade na Tabela 8. A tabela conta apenas os 
artigos de autores que publicaram 4 ou mais artigos na amostra. Foi distribuída uma fração igual do artigo para estado ou país quando o autor declara mais de um vínculo profissional

\section{PRODUTIVIDADE DOS AUTORES DE FINANÇAS NO ENANPAD}

Nesta seção apresentamos uma análise semelhante à anterior, utilizando somente os artigos publicados nos Anais do Enanpad disponíveis na amostra. O quadro é bem diferente do verificado para os periódicos. A primeira grande diferença é que a média de idade dos artigos é bem menor nos Anais, revelando que os autores mais pro- líficos se mantém publicando com freqüência, coisa que não acontece nos periódicos. Embora alguns dos autores mais produtivos nos Anais também estejam entre os mais produtivos na publicação de artigos, vários nomes novos surgem. Há duas possibilidades para essa situação, ou o autor que contribui freqüentemente para os Anais não submete seu trabalho aos periódicos ou ainda não houve tempo para que os trabalhos mais recentes surjam nos periódicos. Outros aspecto marcante na relação dos autores mais produtivos segundo os Anais é que a localização geográfica da vinculação institucional dos autores parece ser mais variada. A Tabela 9 expõe essas informações com mais precisão. Ela mostra somente os autores que publicaram 4 artigos ou mais na amostra selecionada. A idade média dos artigos foi calculada dividindo-se

Tabela 7 - Número de artigos publicados em periódicos nacionais selecionados segundo o vínculo profissional dos autores

\begin{tabular}{|c|c|c|c|c|c|c|c|}
\hline INSTITUIÇÃOO & RAC & RAE & RAUSP & RBE & RBMEC & TOTAL & $\%$ \\
\hline IBMEC/RJ & 3,00 & 6,00 & 7,00 & 0,33 & 38,33 & 54,67 & $26,03 \%$ \\
\hline UFRJ & - & 1,40 & 7,87 & 3,33 & 16,83 & 29,43 & $14,02 \%$ \\
\hline PUC/RJ & 1,00 & 1,00 & 3,33 & 1,00 & 16,80 & 23,13 & $11,02 \%$ \\
\hline USP & - & 6,50 & 10,00 & - & 5,50 & 22,00 & $10,48 \%$ \\
\hline UFRGS & 2,50 & 1,50 & 4,00 & - & 0,50 & 8,50 & $4,05 \%$ \\
\hline CVM/RJ & - & - & - & - & 8,00 & 8,00 & $3,81 \%$ \\
\hline UFSC & 1,00 & 1,00 & 1,50 & 1,00 & 2,50 & 7,00 & $3,33 \%$ \\
\hline UERJ & - & 0,40 & 0,20 & 1,00 & 4,50 & 6,10 & $2,90 \%$ \\
\hline IPEA/RJ & - & 0,40 & 0,87 & 1,33 & 3,33 & 5,93 & $2,83 \%$ \\
\hline NYU/EUA & - & - & 0,33 & - & 5,00 & 5,33 & $2,54 \%$ \\
\hline U. do Minho/Portugal & - & 1,00 & 4,00 & - & - & 5,00 & $2,38 \%$ \\
\hline U. Lancaster/GB & 1,00 & 1,00 & 1,50 & 1,00 & 0,50 & 5,00 & $2,38 \%$ \\
\hline Consultor independente & - & - & - & - & 4,00 & 4,00 & $1,90 \%$ \\
\hline FGV/RJ & - & 0,40 & 0,20 & 1,00 & 2,00 & 3,60 & $1,71 \%$ \\
\hline UFF & - & 0,40 & 0,20 & 1,00 & 2,00 & 3,60 & $1,71 \%$ \\
\hline U. Chicago/EUA & - & - & - & - & 3,50 & 3,50 & $1,67 \%$ \\
\hline Georgetown/EUA & - & - & - & - & 2,50 & 2,50 & $1,19 \%$ \\
\hline UCAM/RJ & - & - & - & - & 2,50 & 2,50 & $1,19 \%$ \\
\hline UCLA/EUA & 2,50 & - & - & - & - & 2,50 & $1,19 \%$ \\
\hline UNR/EUA & - & - & - & - & 2,50 & 2,50 & $1,19 \%$ \\
\hline UFSM & - & - & - & - & 2,00 & 2,00 & $0,95 \%$ \\
\hline BBM & - & - & - & - & 0,80 & 0,80 & $0,38 \%$ \\
\hline IMPA & - & - & - & - & 0,80 & 0,80 & $0,38 \%$ \\
\hline Princeton/EUA & - & - & - & - & 0,80 & 0,80 & $0,38 \%$ \\
\hline Unibanco & - & - & - & - & 0,80 & 0,80 & $0,38 \%$ \\
\hline Total & 11,00 & 21,00 & 41,00 & 11,00 & 126,00 & 210,00 & $100,00 \%$ \\
\hline
\end{tabular}


a idade de cada artigo até 2001 pelo total de artigos publicados. O total de autores é 17 e o de artigos 128.

O vínculo institucional dos autores mais produtivos nos Anais do Enanpad é outro ponto contemplado neste estudo. A UFRGS e a UFRJ lideram a estatística, com mínima vantagem para a primeira. A PUC/RJ aparece em terceiro lugar. Estas três instituições respondem por aproximadamente dois terços dos trabalhos publicados pelos autores com pelo menos 4 trabalhos nos anais da área de

\begin{tabular}{|c|c|c|}
\hline ESTADO OU PAÍS & NÚMERO DE ARTIGOS & $\%$ \\
\hline Rio de Janeiro & 142,67 & 67,94 \\
\hline São Paulo & 22,00 & 10,48 \\
\hline Estados Unidos & 13,83 & 6,59 \\
\hline Rio Grande do Sul & 10,50 & 5,00 \\
\hline Santa Catarina & 7,00 & 3,33 \\
\hline Reino Unido & 5,00 & 2,38 \\
\hline Portugal & 5,00 & 2,38 \\
\hline Não identificado & 4,00 & 1,90 \\
\hline Total & 210,00 & $100,00 \%$ \\
\hline
\end{tabular}

finanças do Enanpad. Surpreende o fato de que instituições importantes na área de administração tenham uma participação pouco expressiva entre os autores mais produtivos da área de finanças. Conforme mostra a Tabela 10. A tabela conta apenas os artigos de autores que publicaram 4 ou mais artigos na amostra. Foi distribuída uma fração igual do artigo para cada instituição quando o autor declara mais de um vínculo profissional.

A Tabela 11 mostra a distribuição geográfica dos autores mais prolíficos em finanças nos Anais do Enanpad. O Rio de Janeiro aparece novamente em primeiro lugar, seguido pelo Rio Grande do Sul e São Paulo. Nota-se a ausência de autores prolíficos de outros estados do país. A tabela conta apenas os artigos de autores que publicaram 4 ou mais artigos na amostra. Foi distribuída uma fração igual do artigo para cada estado ou país quando o autor declara mais de um vínculo profissional.

\section{CONCLUSÃO}

A pesquisa em finanças no Brasil parece ser menos produtiva do que nos EUA, por exemplo. O número de autores com mais de um artigo é menor do que o estimado por modelos bibliométricos. A maioria desses au-

Tabela 9 - Autores que mais publicaram nos Anais do Enanpad

\begin{tabular}{|l|l|c|c|}
\hline \multicolumn{1}{|c|}{ AUTOR } & \multicolumn{1}{|c|}{ VÍNCULOS PROFISSIONAIS RELATADOS } & $\begin{array}{c}\text { No } \\
\text { DE ARTIGOS }\end{array}$ & $\begin{array}{c}\text { IDADE MÉDIA } \\
\text { DOS ARTIGOS }\end{array}$ \\
\hline Eduardo Facó Lemgruber & UFRJ & 22 & 8,32 \\
\hline Jairo Laser Procianoy & UFRGS, USP & 16 & 6,00 \\
\hline Newton C. A. da Costa Júnior & UFSC, UFRJ & 14 & 4,21 \\
\hline Ricardo Pereira Câmara Leal & UFRJ, PUC/RJ, UNR/EUA, Georgetown/EUA & 11 & 7,91 \\
\hline João Luiz Becker & UFRGS & 9 & 12,00 \\
\hline Walter Lee Ness Júnior & PUC/RJ & 8 & 6,63 \\
\hline Manuel José da Rocha Armada & U. do Minho/Portugal & 7 & 4,29 \\
\hline Alfredo Alves de Oliveira Melo & UFMG & 6 & 8,50 \\
\hline Gilberto de Oliveira Kloeckner & UFRGS & 6 & 7,67 \\
\hline Tara Keshar Nanda Baídya & PUC/RJ & 6 & 10,00 \\
\hline Roberto Moreno & PUC/RJ & 5 & 6,20 \\
\hline Alexandre Majola Gava & UFRGS & 4 & 4,75 \\
\hline Carlos Machado dos Santos & UTAD/Portugal & 4 & 3,25 \\
\hline Carlos Patrício Samanez & PUC/RJ, UERJ & 4 & 7,50 \\
\hline Clóvis Graeff & UFRGS & 4 & 12,75 \\
\hline José Paulo Teixeira & PUC/RJ & 4 & 13,25 \\
\hline Richard Saito & FGV/SP & 4 & 1,75 \\
\hline
\end{tabular}


tores está vinculada a instituições do Rio de Janeiro, São Paulo e Rio Grande do Sul, nessa ordem.

Este trabalho analisou uma amostra de artigos publicados no período de 25 anos entre 1974 e 2001 classificados como da área de finanças que aparecem na RAC, RAE, RAUSP, RBE e RBMEC. Os Anais do Enanpad foram analisados separadamente. $O$ periódico que mais contribui para a amostra é a RBMEC, revista com publicação freqüente até meados dos anos 80 . A maioria dos autores mais produtivos publicou em suas páginas nos anos 70 e 80 . Entretanto, muitos deles só publicaram na RBMEC. Mesmo quando se ignora a contribuição dos autores do antigo Ibmec, o Rio de Janeiro ainda aparece como o maior número de autores com 4 ou mais artigos em periódicos. Há evidência de que os autores da USP e

\begin{tabular}{|c|c|c|}
\hline INSTITUIÇÕES & NÚMERO DE ARTIGOS & $\%$ \\
\hline UFRGS & 31,0 & 23,48 \\
\hline UFRJ & 30,5 & 23,11 \\
\hline PUC/RJ & 27,5 & 20,83 \\
\hline USP & 8,0 & 6,06 \\
\hline U. do Minho/Portugal & 7,0 & 5,30 \\
\hline UFSC & 7,0 & 5,30 \\
\hline UFMG & 6,0 & 4,55 \\
\hline $\mathrm{FGV} / \mathrm{SP}$ & 4,0 & 3,03 \\
\hline UTAD/Portugal & 4,0 & 3,03 \\
\hline Georgetown/EUA & 2,5 & 1,89 \\
\hline UNR/EUA & 2,5 & 1,89 \\
\hline UERJ & 2,0 & 1,52 \\
\hline Total & 132,0 & 100,00 \\
\hline
\end{tabular}

Tabela 11 - Número de artigos publicados nos Anais do Enanpad de acordo com o estado ou país dos autores

\section{INSTITUICÕ̃ES \\ NÚMERO DE ARTIGOS}

Rio de Janeiro

Rio Grande do Sul

São Paulo

Portugal

Santa Catarina

Minas Gerais

Estados Unidos

Total

60

45,45

23,48

31

9,09

12

8,33

11

5,30

7

4,55

3,79
100,00 da FGV-EAESP utilizam a revista de suas respectivas instituições, RAUSP e RAE, com mais frequência. A idade média dos artigos publicados pela maioria dos autores mais prolíficos é alta.

Quando se examinam os Anais do Enanpad, a renovação pode estar a caminho. A idade média dos artigos dos autores com 4 ou mais artigos nos Anais é bem menor e há vários nomes novos. Os vínculos institucionais dos autores prolíficos na área de finanças do Enanpad são bem variados. A UFRGS aparece com destaque, seguida da UFRJ e da PUC/RJ. O Rio de Janeiro aparece novamente com o maior número de artigos e de autores prolíficos, seguido do Rio Grande do Sul e de São Paulo. Nota-se a ausência de autores prolíficos que não atuem nas regiões Sul e Sudeste.

Artigo recebido em 23/04/2002. Aprovado em 08/10/2002.

\section{Notas}

Os autores agradecem a Túlio Ribeiro, que tornou o banco de dados utilizado uma realidade, às instituições que forneceram dados sobre suas teses e publicações, a dois revisores anônimos da RAE e ao CNPq pelo apoio. Todos os erros são de nossa inteira responsabilidade.

1. O antigo Ibmec, o Instituto Brasileiro de Mercado de Capitais, era uma instituição sem fins lucrativos que realizava estudos sobre o mercado financeiro no Brasil e, mais tarde, passou a realizar programas educacionais. O Ibmec atual é uma empresa fundada em 1999 que absorveu as atividades educacionais do antigo Ibmec.

2. O banco de dados está disponivel para consulta em www.coppead.ufrj.br/ cefin/busca.html. Além das referências dos artigos das revistas já mencionadas o banco de dados conta com referências de artigos selecionados da Resenha BM\&F e de dissertações e teses, apesar desses dois últimos não terem sido utilizados no estudo, de algumas instituições de ensino nacionais. O banco de dados não contém artigos para download, apenas referências bibliográficas.

3. Para o Enanpad de 2000 havia 43 apresentações em marketing contra 27 em finanças.

\section{Referências bibliográficas}

ALEXANDER, Jr., JOHN C.; MABRY, Rodney H. Relative significance of journals, authors, and articles cited in financial research. Journal of Finance, v. 49, n. 2, p. 697-712, 1994.

AZZONI, Carlos R. "Clássicos" da literatura econômica brasileira: trabalhos e autores mais citados nas nossas revistas acadêmicas. Economia Aplicada, v. 2, n. 4, p. 771-780, 1998

Desempenho das revistas e dos departamentos de economia brasileiros segundo publicações e citações recebidas no Brasil. Economia Aplicada, v. 4, n. 4, p. 786-822, 2000. 
BERTERO, Carlos O.; KEINERT, Tânia M.M. A evolução da análise organizacional no Brasil (1961-93). Revista de Administração de Empresas, v. 34, n. 3, p. 81-90, 1994.

BIGNETTI, Luiz Paulo; PAIVA, Ely Laureano. Ora (direis) ouvir estrelas! estudo das citações de autores de estratégia na produção acadêmica brasileira. Revista de Administração Contemporânea, v. 6, n. 1, p. 105-125, 2002.

BOROKHOVICH, Kenneth A.; BRICKER, Robert J.; SIMKINS, Betty J. Journal communication and influence in financial research. Journal of Finance, v. 49, n. 2, p. 713-725, 1994.

CHUNG, Kee H.; COX, Raymond A. K. Patterns of productivity in the finance literature: a study of the bibliometric distributions. Journal of Finance, v. 45, n. 1, p. 301-309, 1990.

FARIA, João R. The research output of academic economists in Brazil. Economia Aplicada, v. 4, n. 1, p. 95-111, 2000.

FROEMMING, Lurdes M. S.; LUCE, Fernando B.; PERIN, Marcelo G.; SAMPAIO, Cláudio H.; BEBER, Sedinei J. N.; TREZ, Guilherme. Inventário de artigos científicos na área de marketing no Brasil. Revista de Administração Contemporânea, v. 4, n. 2, p. 159-173, 2000.

KOLJATIC, Mladen; SILVA, Mónica. Authorship characteristics of LatinAmerican publications in economics and business administration: an exploratory assessment. Revista Abante, v. 4, n. 1, p. 83-94, 2001.

MITENKO, Graham R.; DIAMOND, Jeanette M. Publication policies and practices of major finance journals: a survey of editors. Financial Practice and Education, v. 4, n. 2, p. 88-93, 1994.

NESS JR., Walter L. Apresentação. In: ENCONTRO ANUAL DA ASSOCIAÇÃO NACIONAL DOS PROGRAMAS DE PÓS-GRADUAÇÃO EM ADMINISTRAÇÃO, 22, 1998, Foz do Iguaçu.

De OLIVEIRA, Ernani T. Apresentação. In: ENCONTRO ANUAL DA ASSOCIAÇÃO NACIONAL DOS PROGRAMAS DE PÓS-GRADUAÇÃO EM ADMINISTRAÇÃO, 4, 1980, Angra dos Reis.
POWERS, Thomas L.; SWAN, John E.; PATTON, John F. Career research productivity patterns of marketing academicians. Journal of Business Research, v. 42, n. 1, p. 75-86, 1998

VASCONCELLOS, Eduardo. Apresentação. In: ENCONTRO ANUAL DA ASSOCIAÇÃO NACIONAL DOS PROGRAMAS DE PÓS-GRADUAÇÃO EM ADMINISTRAÇÃO, 5, 1981. Anais ... ANPAD, 1981, p. 3-4.

VERGARA, Sylvia C.; CARVALHO, JR., Dourival S. Nacionalidade dos autores referenciados na literatura brasileira sobre organizações. In: $19^{\circ}$ ENCONTRO ANUAL DA ASSOCIAÇÃO NACIONAL DOS PROGRAMAS DE PÓS-GRADUAÇÃO EM ADMINISTRAÇÃO, 1995, Angra dos Reis. Anais ... Angra dos Reis : ANPAD, 1995, p. 169-188.

VIEIRA, Francisco G. D. Por quem os sinos dobram? Uma Análise da Publicação Científica na Área de Marketing do Enanpad. In: ENCONTRO ANUAL DA ASSOCIAÇÃO NACIONAL DOS PROGRAMAS DE PÓS-GRADUAÇÃO EM ADMINISTRAÇÃO, 22, 1998, Foz do Iguaçu. Anais ... Foz do Iguaçu: ANPAD, 1998.

Narciso sem espelho: a publicação científica de marketing no Brasil nos anos 90. Mimeo, Universidade Estadual de Maringá, 2000.

ZIVNEY, Bertin. Publish of perish: what the competition is really doing. Journal of Finance, v. 47, n. 1, p. 295-329, 1992.

ZIVNEY, Terry L.; CALLAWAY, Richard E.; BERTIN, William J. The publication experience of meeting presentations. Financial Practice and Education, v. 4, n. 2, p. 94-98, 1994.

ZIVNEY, Terry L.; REICHENSTEIN, William. The pecking order in finance journals. Financial Practice and Education, v. 4, n. 2, p. 77-87, 1994.

\section{Ricardo Pereira Câmara Leal}

Diretor e Professor de Finanças do Instituto COPPEAD de Administração, UFRJ

Interesses de pesquisa: governança corporativa, análise de investimentos, custo de capital.

E-mail: rleal@coppead.ufrj.br

Endereço: Caixa Postal 68514 - Rio de Janeiro, RJ 21945-970.

\section{Jefferson de Oliveira}

Atuário na área de controladoria da REFER - Fundação Rede Ferroviária de Seguridade Social

E-mail: jefferson@refer.com.br

Endereço: R. da Quitanda, 173 - Rio de Janeiro, RJ 20091-005

\section{Aline Feldman Soluri}

Estagiária na Área de Risco do Mellon Brascan DTVM e Bolsista de Iniciação Científica CNPq

E-mail: aline@mellonbrascan.com.br

Endereço: R. da Assembléia 10, 17ํandar - Rio de Janeiro, RJ 20011-000 
Apêndice - Levantamento de publicações na área de finanças em língua inglesa dos autores prolíficos

O levantamento foi feito a partir de solicitações feitas aos próprios autores para que listassem suas publicações em periódicos de língua inglesa com sistema blind review e de pesquisa na Internet e em bases de dados como a PROQUEST, ECONLIT e ECONBASE da Elsevier. Nem todos os autores têm publicações e alguns não foram localizados. A listagem é apenas ilustrativa da atividade dos autores e não pretende ser exaustiva.

\begin{tabular}{|c|c|c|}
\hline AUTOR & № DE PUBLICAÇÕES & PERIÓDICO (E ANO DE PUBLICAÇÃO) \\
\hline Antônio Marcos Duarte Jr. & 13 & $\begin{array}{l}\text { Journal of Portfolio Management }(1997,1999) \\
\text { Emerging Markets Quarterly }(1997(2)) \\
\text { Derivatives Quarterly }(1997,1998) \\
\text { Journal of Fixed Income }(1998,2000) \\
\text { Journal of Derivatives }(1998,1999(2)) \\
\text { Journal of Financial Engineering (1999) } \\
\text { Journal of Risk (1999) }\end{array}$ \\
\hline Clóvis de Faro & 3 & J. of Financial and Quantitative Analysis (1975 (2) e 1978) \\
\hline Jairo L. Procianoy & 2 & $\begin{array}{l}\text { Latin American Business Review (2001) } \\
\text { International Review of Financial Analysis (2001) }\end{array}$ \\
\hline João L. Becker & 3 & $\begin{array}{l}\text { Management Science (1987) } \\
\text { Journal of Risk and Uncertainty (1989) } \\
\text { Integrated Manufacturing Systems (1999) }\end{array}$ \\
\hline Newton C. A. Costa Jr. & 2 & $\begin{array}{l}\text { Journal of Banking and Finance (1994) } \\
\text { Emerging Markets Quarterly (1998) }\end{array}$ \\
\hline Ney R. O. Brito & 3 & $\begin{array}{l}\text { Jorunal of Finance (1977 e 1978) } \\
\text { Journal of Banking and Finance (1979) }\end{array}$ \\
\hline Pedro C. Mello & 1 & Journal of Economics and Business (2001) \\
\hline Ricardo P. C. Leal & 13 & $\begin{array}{l}\text { Financial Management (1993) } \\
\text { Journal of Emerging Markets (1996, 1999, 2001) } \\
\text { International Journal of Finance (1998 e 2000) } \\
\text { Emerging Markets Quarterly (1998 e 2000) } \\
\text { Journal of Banking and Finance (1999) } \\
\text { Journal of Financial and Quantitative Analysis (1999) } \\
\text { International Review of Financial Analysis (2001 (2)) } \\
\text { Corporate Governance (2002) }\end{array}$ \\
\hline Rubens Famá & 1 & Journal of Emerging Markets (1997) \\
\hline Walter L. Ness Jr. & 7 & $\begin{array}{l}\text { Financial Management (1972) } \\
\text { Journal of International Business Studies (1975) } \\
\text { Financial Markets, Institutions \& Instruments (1975) } \\
\text { Journal of Finance (1976) } \\
\text { Journal of Banking and Finance (1977) } \\
\text { Quarterly Review of Economics and Finance }(2000,2002)\end{array}$ \\
\hline
\end{tabular}

